\title{
Use of Brentuximab Vedotin in a Non-HIV Patient with Primary Effusion Lymphoma
}

\author{
$\underline{\text { Joana Ferra }^{1}}$, Filipa Mousinho $^{2}$, Francisca Miranda ${ }^{2}$, Sandra Andréé ${ }^{1}$, Celina Afonso ${ }^{2}$, Fernando Nogueira $^{1}$ \\ ${ }^{1}$ Pneumology Department, Hospital Egas Moniz, Centro Hospitalar Lisboa Ocidental, E.P.E., Portugal \\ ${ }^{2}$ Hematology Department, Hospital São Francisco Xavier, Centro Hospitalar Lisboa Ocidental, E.P.E., Portugal
}

\section{Doi: 10.12890/2021_002345- European Journal of Case Reports in Internal Medicine - ๑ EFIM 2021}

Received: 01/02/2021

Accepted: 02/02/2021

Published: $22 / 02 / 2021$

How to cite this article: Ferra J, Mousinho F, Miranda F, André S, Afonso C, Nogueira F. Use of brentuximab vedotin in a non-HIV patient with primary effusion lymphoma. EJCRIM 2021;8: doi:10.12890/2021_002345.

Conflicts of Interests: The Authors declare that there are no competing interests.

Acknowledgements: The authors would like to thank Dr. Lucília Monteiro (Anatomical Pathology Department, Hospital Egas Moniz, Centro Hospitalar Lisboa Ocidental, E.P.E., Portugal) for providing the images in Figure 2.

This article is licensed under a Commons Attribution Non-Commercial 4.0 License

\section{ABSTRACT}

Primary effusion lymphoma is a rare and aggressive large B-cell lymphoma presenting as malignant effusion with poor prognosis. Although it is more prevalent among HIV patients, it has also been described in non-HIV immunocompromised individuals. Given its rarity, there are no large randomized trials regarding the best therapeutic option. The choice of therapy is based on retrospective studies, case reports and preclinical data. We present the case of a non-HIV patient with relapsed disease after treatment with CHOP who was then successfully treated with brentuximab vedotin, achieving complete remission.

\section{LEARNING POINTS}

- Primary effusion lymphoma is a rare entity in non-HIV patients, so there are no clinical trials regarding therapeutic options or management decisions.

- Targeted therapy with brentuximab vedotin has been used in other CD30 positive malignancies and in HIV-related primary effusion lymphoma with good outcomes.

- We present the case of a non-HIV patient with primary effusion lymphoma who was successfully treated with brentuximab vedotin, which highlights the potential role of a new therapeutic approach in this subgroup of patients.

\section{KEYWORDS}

Primary effusion lymphoma, brentuximab vedotin, non-HIV

\section{CASE DESCRIPTION}

A 77-year-old male patient, a non-smoker, with a medical history of arterial hypertension and left shoulder arthroplasty was admitted to hospital in November 2018 due to cardiac tamponade. He underwent pericardiocentesis with drainage of $1250 \mathrm{ml}$ of serohematic fluid: both cytological and microbiological analyses were negative, including a nucleic acid amplification test (NAAT) for Mycobacterium tuberculosis; immunophenotypic analysis was not performed. A post-procedure echocardiogram did not reveal any alterations, there was no recurrence of pericardial effusion and the patient was discharged. Six months later he was admitted to the hospital with worsening dyspnoea. He denied fever, cough, sputum, chest pain, lower extremity swelling, or any other symptoms. Laboratory analyses at admission were normal. HIV and hepatitis testing was negative. HHV-8 serology testing was not available. Chest radiography revealed a right pleural effusion that was not present at the previous hospital discharge (Fig. 1A). Thoracentesis was performed. Cytochemical findings revealed the characteristics of 
an exudate, microbiological analysis, including NAAT for M. tuberculosis, was negative and cytological examination revealed 13,590 cells/ ul: neutrophils $4 \%$, lymphocytes $6 \%$, eosinophils $5 \%$, monocytes/macrophages $17 \%$, and other cells $68 \%$ (cells of lymphoid morphology, irregular nucleus). An extensive immunophenotypic analysis was performed which found $83.6 \%$ of cells were pathological, with a phenotype compatible with primary effusion lymphoma (positive for CD30, CD38, CD4, CD123, HLA-DR, CD45 (weak); negative for CD2, CD3, CD5, CD7, CD8, CD19, CD20, CD56, CD33, CD34, CD71, CD138, CD326). Pleural fluid cytology demonstrated atypical lymphoid cells, compatible with non-Hodgkin B-large cell lymphoma, primary effusion lymphoma (CD30+, HHV8+, CD20-, CD3-) (Fig. 2).

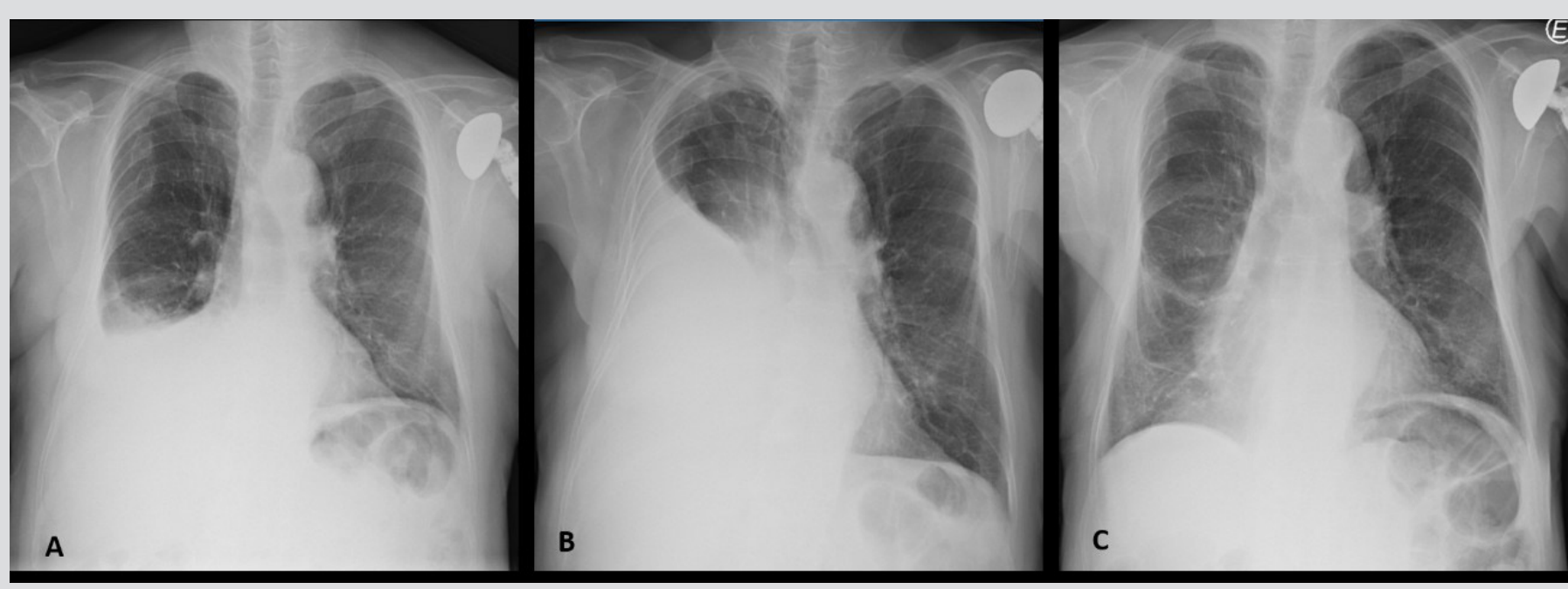

Figure 1. Radiological evolution of a patient with pleural effusion due to primary effusion lymphoma. (A) At the time of diagnosis; (B) after four cycles of CHOP; (C) after 16 cycles of brentuximab vedotin
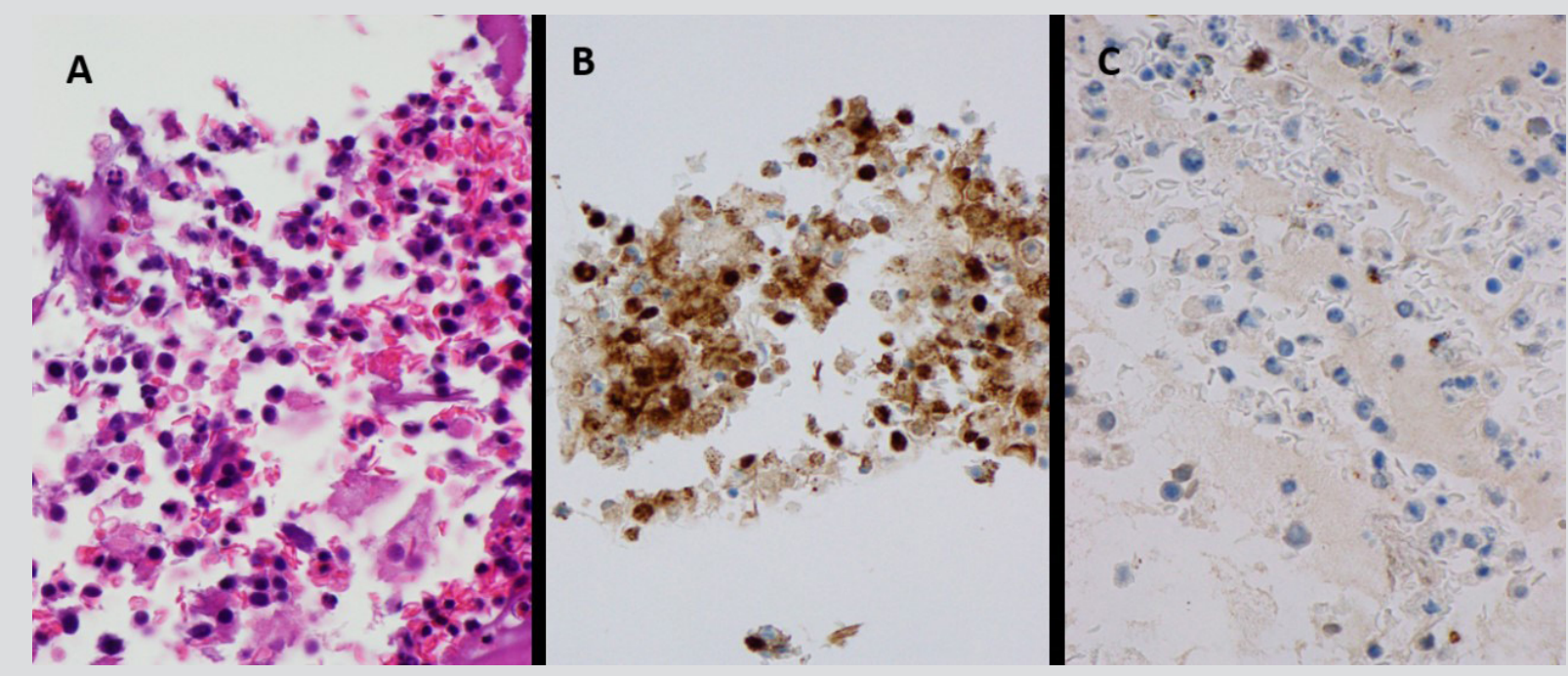

Figure 2. (A) Large, atypical lymphoid cells, with plasmacytoid morphology and irregular nucleus compatible with non-Hodgkin large B-cell lymphoma, primary effusion lymphoma. (B) Lymphoid cells were positive for HHV-8 and CD30. (C) Lymphoid cells were negative for CD2O and CD3

Cervical-thoracic-abdominal-pelvic computed tomography (CT) excluded nodal or other organ involvement by the lymphomatous process. Bone marrow biopsy showed the presence of the three blood cell lines without significant changes and no pathological infiltrates.

The consistent morphology and immunophenotypic characteristics and clinical presentation led to the diagnosis of primary effusion lymphoma. The patient was started on chemotherapy with CHOP (cyclophosphamide, doxorubicin, vincristine, prednisolone), showing progression by the fourth cycle, and needed recurrent thoracentesis for symptomatic relief (Fig. 1B). Since the lymphoma was CD30+, he was then started on second-line therapy with brentuximab vedotin and achieved complete remission by the seventh cycle. 
He has now completed 16 cycles of brentuximab vedotin and given the significant clinical response (Fig. 1C), with no toxicities, it was decided to extend the treatment until disease progression or unacceptable toxicity.

\section{DISCUSSION}

Primary effusion lymphoma (PEL) is a rare and aggressive large B-cell lymphoma presenting as malignant effusions ${ }^{[1]}$. It was first described in HIV patients, accounting for approximately 4\% of HIV-associated lymphomas and less than $1 \%$ of non-HIV lymphomas ${ }^{[1]}$. In non-HIV patients, it is more prevalent in immunocompromised patients such as recipients of a solid organ transplant, patients with hepatic cirrhosis, or in older males in areas of high prevalence of HHV-8 infection such as the Mediterranean region ${ }^{[2,3]}$.

PEL clinical presentation depends on disease location and the extent of the effusion. The most common locations are the pleural space, peritoneal cavity, pericardial space and joint spaces, but some patients may present other organ involvement in late stages of the disease ${ }^{[1]}$. PEL diagnosis is based on the morphological and immunophenotypic characteristics of malignant cells that are obtained from the drainage of cavity fluid. PEL is also known to be associated with HHV-8 infection in the nuclei of malignant cells ${ }^{[1]}$. They are usually CD45+, proving a lymphoid origin, but do not show typical B-cell (including surface and cytoplasmic immunoglobulin, CD19, CD20, CD79a) or T-cell immunophenotypic characteristics (CD3, CD4, CD8) ${ }^{[1]}$. PEL is usually CD30+, CD38+ and CD138+ [1]. The differential diagnosis includes other non-Hodgkin lymphomas that present with lymphomatous effusions ${ }^{[2]}$.

PEL has a poor prognosis and its optimal therapy has not yet been established ${ }^{[2]}$. Given the low incidence of PEL, there are no randomized clinical trials on the best therapy and management decisions. The choice of therapy is usually based on retrospective studies, case reports and preclinical data ${ }^{[1]}$. Usually, first-line chemotherapy includes a combination of cyclophosphamide, doxorubicin, vincristine and prednisone regimens. Although there is an initial response, most patients have disease relapse within 6-8 months or do not tolerate these regimens due to toxicity ${ }^{[1]}$. In relapsed and refractory disease, treatment options will depend upon performance status and comorbidities ${ }^{[1,2]}$. Multiple options have been tried and there are some case reports describing variable responses and outcomes, such as stem cell transplant, radiation and antiviral agents targeted against HHV-8 (for example ganciclovir and foscarnet) ${ }^{[1]}$.

Some targeted therapies have been used with promising results such as brentuximab vedotin, an antibody drug conjugated against CD30, currently approved in previously treated classic Hodgkin lymphoma and anaplastic large cell lymphoma ${ }^{[1]}$. As PEL is usually CD30+, in preclinical data the use of brentuximab vedotin led to apoptosis of malignant cells and induced tumour regression and prolonged survival of mice with PEL ${ }^{[4]}$. There are a few case reports of HIV-related PEL treated with brentuximab vedotin with promising results after disease relapse ${ }^{[5]}$. In our case, we have presented a non-HIV patient with PEL who achieved complete remission with brentuximab vedotin, which highlights the potential role of a new therapeutic approach for non-HIV patients with PEL.

\section{REFERENCES}

1. Narkhede M, Arora S, Ujjani C. Primary effusion lymphoma: current perspectives. Onco Targets Ther 2018;11:3747-3754

2. Okada S, Goto H, Yotsumoto M. Current status of treatment for primary effusion lymphoma. Intractable Rare Dis Res 2014;3(3):65-74.

3. Klepfish A, Sarid R, Shtalrid M, Shvidel L, Berrebi A, Schattner A. Primary effusion lymphoma (PEL) in HIV-negative patients - a distinct clinical entity. Leuk Lymphoma 2001;41(34):439-443.

4. Bhatt S, Ashlock BM, Natkunam Y, Sujoy V, Chapman JR, Ramos JC, et al. CD30 targeting with brentuximab vedotin: a novel therapeutic approach to primary effusion lymphoma. Blood 2013;122(7):1233-1242.

5. Chang VA, Wang HY, Reid EG. Activity of brentuximab vedotin in AIDS-related primary effusion lymphoma. Blood 2019;3(5):766-768. 